\title{
Implicancia de la radiografía con tracción y rotación interna en la evaluación de las fracturas de fémur proximal
}

\author{
Fernando M. Bidolegui, Sebastián Pereira, Gabriel Vindver \\ Servicio de Ortopedia y Traumatología, Hospital Sirio Libanés, ECICARO, Ciudad Autónoma de Buenos Aires, Argentina
}

\begin{abstract}
RESUMEN
Introducción: La correcta valoración de las radiografías cuando ingresa un paciente con una fractura de fémur proximal tiene una implicancia directa en el tratamiento propuesto. Materiales y Métodos: Se evaluó consecutivamente a 100 pacientes con diagnóstico de fractura de fémur proximal. Se tomaron radiografías de pelvis de frente, de la cadera afectada de frente y de la cadera afectada de frente con tracción y rotación interna. Se comparó la clasificación realizada por residentes con la clasificación de los médicos senior, todos utilizaron las tres radiografías para evaluar las fracturas. Resultados: El resultado global de concordancia entre la clasificación inicial de los residentes con la de los médicos senior fue del 68,9\%. Cuando los médicos residentes utilizaron la radiografía con tracción y rotación interna, la concordancia aumentó al 78,75\%. Cincuenta y una respuestas cambiaron con respecto a la clasificación inicial. De estas, en $42(82,4 \%)$ casos, la clasificación inicial era incorrecta y cambió a una correcta. En 9 $(17,6 \%)$ casos, la clasificación inicial era correcta y cambió a una incorrecta. Conclusiones: La radiografía con tracción y rotación interna es un estudio simple, de bajo costo y bien tolerado por el paciente que facilita la correcta interpretación de las fracturas de fémur proximal, lo que tiene un impacto directo en la indicación del tratamiento y su resultado final.
\end{abstract}

Palabras clave: Fracturas; fémur proximal; clasificación; radiografía con tracción.

Nivel de Evidencia: IV

Implication of Internal Rotation Traction Radiography in Proximal Femoral Fracture Evaluation

\begin{abstract}
Introduction: The correct assessment of radiographs at the time of admission of a patient with proximal femoral fracture has a direct effect on the choice of treatment. Materials and Method: We consecutively evaluated 100 patients with a diagnosis of proximal femoral fracture. Antero-posterior pelvic radiographs (A-P), A-P radiographs of the affected hip, and internal rotation traction radiographs of the affected hip were taken. A comparison was made between the classifications made by residents and the classification of senior doctors, who used the 3 radiographs to classify all fractures. Results: The overall agreement score between the initial classification of residents and that of senior doctors was $68.9 \%$. When the resident physicians used internal rotation traction radiography, agreement increased to $78.75 \% .51$ responses changed with respect to the initial classification. Of these, in $42(82.4 \%)$ cases the initial classification was incorrect and changed to a correct classification. While in $9(17.6 \%)$ cases the initial classification was correct and changed to an incorrect one. Conclusion: Internal rotation traction radiography is a simple, low-cost study that is well-tolerated by the patient and facilitates correct interpretation of proximal femoral fractures with a direct impact on the choice of treatment and its outcome.
\end{abstract}

Key words: Fractures; proximal femur; classification; traction radiography.

Level of Evidence: IV

Recibido el 4-5-2020. Aceptado luego de la evaluación el 6-7-2020 • Dr. FERNANDO M. BIDOLEGUI • fbidolegui@gmail.com (ID) https://orcid.org/0000-0002-0502-2300

Cómo citar este artículo: Bidolegui FM, Pereira S, Vindver G. Implicancia de la radiografía con tracción y rotación interna en la evaluación de las fracturas de fémur proximal. Rev Asoc Argent Ortop Traumatol 2021;86(1):39-43. https://doi.org/10.15417/issn. 1852-7434.2021.86.1.1112 


\section{INTRODUCCIÓN}

Las fracturas de fémur proximal son lesiones comunes en los pacientes añosos y su incidencia está en aumento. ${ }^{1}$ La elección del tratamiento más adecuado es fundamental para disminuir las tasas de morbimortalidad asociadas, así como los costos del sistema de salud. ${ }^{1}$ La correcta valoración de las radiografías cuando ingresa un paciente con fractura de fémur proximal tiene una implicancia directa en la toma de decisiones sobre su tratamiento. El objetivo de este estudio es demostrar la utilidad de la radiografía con tracción y rotación interna tomada al ingreso del paciente en la evaluación de las fracturas de fémur proximal.

\section{MATERIALES Y MÉTODOS}

Se evaluó, en forma retrospectiva, una serie consecutiva de pacientes $>65$ años con diagnóstico de fractura de fémur proximal que fueron operados por los médicos del Servicio de Ortopedia y Traumatología de nuestro Hospital, entre enero de 2018 y enero de 2020. Se excluyó a los pacientes con fracturas subtrocantéricas y fracturas patológicas. La serie quedó conformada por 100 pacientes. Se tomaron radiografías de pelvis de frente, radiografías de la cadera afectada de frente y radiografías de la cadera afectada de frente con tracción y rotación interna. Cuando se consideró necesario, se completó la evaluación con una tomografía computarizada. Para obtener las radiografías con tracción, se coloca al paciente en decúbito supino, mientras el médico realiza una tracción suave y progresiva desde el tobillo, a la vez, que le imprime una rotación interna a la extremidad hasta llevar la rótula al cenit. Luego de tomar la imagen se libera la tracción suavemente. En ocasiones, puede ser necesaria la ayuda de un asistente para realizar contratracción.

Las radiografías fueron evaluadas por médicos senior (S.P., F.B. y G.V.) quienes clasificaron las fracturas observando las tres radiografías y las imágenes tomográficas, si correspondía. Se utilizaron la clasificación de Garden ${ }^{2}$ para las fracturas de cuello femoral y los criterios de inestabilidad descritos por Evans ${ }^{3}$ (conminución, compromiso de la pared posteromedial, extensión subtrocantérica y verticalidad del trazo) para las fracturas intertrocantéricas. Las respuestas posibles eran: 1) fractura de cuello femoral no desplazada (Garden tipos I y II), 2) fractura de cuello femoral desplazada (Garden tipos III y IV), 3) fractura intertrocantérica estable y 4) fractura intertrocantérica inestable (al menos, un criterio de inestabilidad). Las respuestas de los médicos senior fueron consideradas como de referencia. Luego fueron comparadas con las respuestas dadas por cuatro médicos residentes de los primeros años, quienes, de forma independiente y sin información sobre el paciente, agruparon las fracturas en los cuatro grupos posibles utilizando las radiografías de pelvis de frente y las radiografías de la cadera afectada de frente, en una primera ronda (400 respuestas). En una segunda ronda (400 respuestas) con un intervalo de dos semanas respecto de la anterior, los mismos cuatro residentes bajo las mismas condiciones clasificaron las mismas fracturas en los cuatro grupos posibles observando las radiografías de la cadera afectada de frente con tracción y rotación interna.

\section{RESULTADOS}

La distribución de las 100 fracturas según la respuesta de los médicos senior fue la siguiente: fracturas de cuello femoral no desplazadas (8 casos), fracturas de cuello femoral desplazadas (24 casos), fracturas intertrocantéricas estables ( 25 casos) y fracturas intertrocantéricas inestables (43 casos).

La concordancia entre la clasificación inicial de los residentes (ronda 1) con la de los médicos senior fue del $68,75 \%$ ( 275 respuestas correctas). Cuando los médicos residentes utilizaron la radiografía con tracción y rotación interna (ronda 2), la concordancia global aumentó al 78,75\% (315 respuestas correctas) (Tabla 1).

Tabla 1. Efecto de la radiografía con tracción y rotación interna en la clasificación de los médicos residentes

\begin{tabular}{|l|c|c|}
\hline & Radiografia anteroposterior & $\begin{array}{c}\text { Radiografia anteroposterior con tracción } \\
\text { y rotación interna }\end{array}$ \\
\hline Clasificación correcta & 275 & 326 \\
\hline Clasificación incorrecta & 125 & 74 \\
\hline Total de respuestas & 400 & 400 \\
\hline
\end{tabular}


Un total de 51 respuestas cambiaron con respecto a la clasificación inicial cuando se tuvo en cuenta la radiografía con tracción y rotación interna. En 42 de las 51 respuestas $(82,4 \%)$, la clasificación inicial era incorrecta y cambió a una clasificación correcta. Los cambios correctos en las respuestas fueron los siguientes: de una fractura intertrocantérica estable a una fractura intertrocantérica inestable (24 casos); de una fractura de cuello femoral desplazada a una fractura intertrocantérica estable (12 casos) y de una fractura intertrocantérica estable a una fractura de cuello femoral desplazada (6 casos) (Tabla 2).

Tabla 2. Cambios correctos en la clasificación de los residentes con la radiografía con tracción y rotación interna

\begin{tabular}{|l|c|}
\hline Cambio en la clasificación & Cantidad de respuestas \\
\hline De fractura intertrocantérica estable a fractura intertrocantérica inestable & 24 \\
\hline De fractura de cuello femoral desplazada a fractura intertrocantérica estable & 12 \\
\hline De fractura intertrocantérica estable a fractura de cuello femoral desplazada & 6 \\
\hline Total de cambios correctos & 42
\end{tabular}

Por otro lado, nueve $(17,6 \%)$ de las 51 respuestas cambiaron de una clasificación inicial correcta a una incorrecta al evaluar la radiografía con tracción y rotación interna: siete casos de fracturas intertrocantéricas inestables cambiaron a fracturas intertrocantéricas estables, un caso cambió de fractura intertrocantérica estable a fractura de cuello femoral desplazada (Figura) y un caso cambió de fractura de cuello femoral desplazada a fractura de cuello femoral no desplazada (Tabla 3).

Tabla 3. Cambios incorrectos en la clasificación con la radiografía con tracción y rotación interna

\section{Cambio en la clasificación}

De fractura intertrocantérica inestable a fractura intertrocantérica estable

De fractura de cuello femoral desplazada a fractura de cuello femoral no desplazada

De fractura intertrocantérica estable a fractura de cuello femoral desplazada

Total de cambios incorrectos
Cantidad de respuestas

7
1
1
9

\section{DISCUSIÓN}

La correcta valoración de las radiografías cuando ingresa un paciente con una fractura de fémur proximal tiene una implicancia directa en la indicación del tratamiento. En general, cuando llega un paciente con una fractura de cadera, tiene dolor y el miembro afectado acortado y en rotación externa. Esto asociado a la anteversión natural del cuello femoral dificulta la correcta evaluación de la fractura. La radiografía con tracción y rotación interna despliega el cuello femoral y evita la superposición de los fragmentos, lo que facilita su interpretación. ${ }^{8}$ Esta simple maniobra, de tracción y rotación interna, cuando se realiza suavemente e incrementando de a poco la fuerza de tracción, es bien tolerada por el paciente y no requiere de ningún tipo de sedación.

Las fracturas de cuello femoral suelen clasificarse según la clasificación de Garden. ${ }^{2}$ Sin embargo, algunos autores han documentado una baja reproducibilidad interobservador con esta clasificación. ${ }^{3}$ En el mismo sentido, diferentes autores resaltan la misma dificultad en la clasificación inicial de las fracturas intertrocantéricas de cadera. ${ }^{4-7}$ Los resultados de nuestro estudio resaltan la variabilidad interobservador en la clasificación de las fracturas de fémur proximal. La concordancia entre la clasificación de los médicos residentes utilizando las radiografías sin tracción con la clasificación establecida por los médicos senior fue del $68,75 \%$. Ahora cuando los residentes utilizaron la radiografía con tracción y rotación interna, la concordancia aumentó al 78,75\% . 
Si analizamos los cambios en las respuestas, en 18 de los 42 casos en los que se cambió la respuesta por una correcta, esto hubiese significado una modificación en la indicación quirúrgica. Doce de estos 18 casos fueron clasificados inicialmente como fracturas de cuello femoral desplazadas que cambiaron a fracturas intertrocantéricas estables, esto hubiese significado, por la edad de nuestra serie, tratar con una artroplastia de cadera una fractura intertrocantérica estable. En los seis casos restantes, la modificación fue de una fractura intertrocantérica estable a una fractura de cuello femoral desplazada, lo que hubiese significado tratar con una reducción y osteosíntesis una fractura de cuello femoral en un paciente añoso. En 24 de los 42 casos restantes, el cambio en la clasificación de una fractura estable a una inestable no hubiese tenido repercusión en la elección del implante, porque, en nuestro Servicio, el implante de elección para las fracturas intertrocantéricas de cadera sean estables o inestables es el clavo de fémur proximal. Sin embargo, en aquellos Servicios donde se utiliza un clavo compresivo deslizante (DHS) para las fracturas estables y un clavo de fémur proximal para las inestables, este cambio hubiese significado una modificación en la selección del implante.

Al evaluar los nueve casos $(17,6 \%)$ en los que la radiografía con tracción y rotación interna cambió una respuesta correcta por una incorrecta, solo en dos pacientes, el cambio de respuesta hubiese tenido repercusión en la indicación del tratamiento: en un caso, porque la modificación fue de una fractura intertrocantérica estable a una fractura de cuello femoral desplazada (Figura) y, en el otro, de una fractura de cuello femoral desplazada a una fractura de cuello femoral no desplazada.
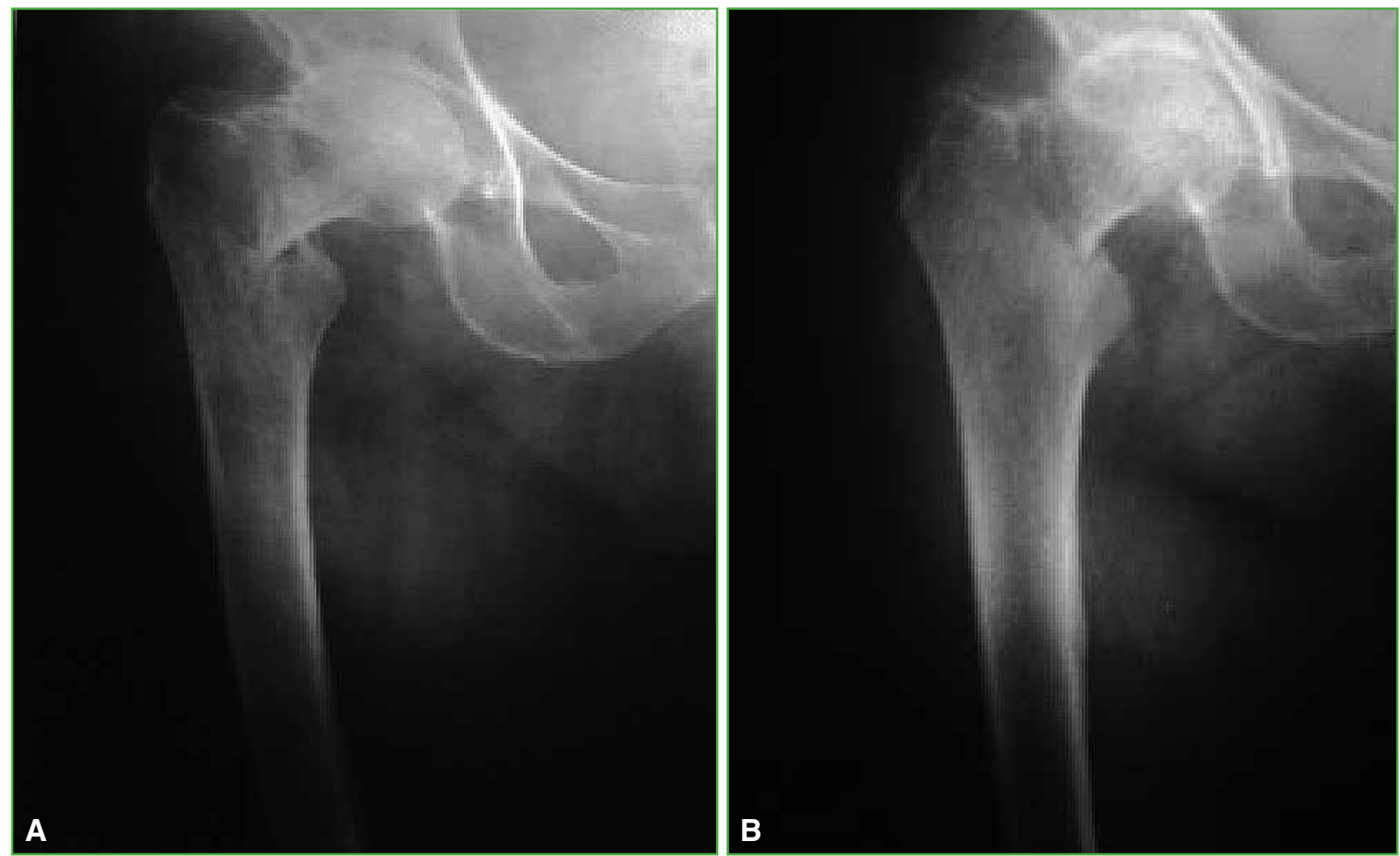

Figura. A. Radiografía anteroposterior sin tracción. Se observa una aparente fractura lateral desplazada. B. Radiografía con tracción y rotación interna. Se observa una fractura medial.

Hasta nuestro conocimiento pocos son los estudios que analizan la utilidad de las radiografías con tracción en la evaluación inicial de las fracturas de fémur proximal. En 1976, Wiltse ${ }^{9}$ fue el primero en marcar la utilidad de la radiografía con tracción y rotación interna para aumentar la sensibilidad de la detección de fracturas ocultas de cadera. 
En un estudio más reciente, Koval y cols..$^{10}$ evaluaron la utilidad de la radiografía con tracción comparando la concordancia en la clasificación de 47 radiografías con fractura de cadera entre los médicos residentes y los médicos senior. La concordancia fue del 71,9\% sin la radiografía con tracción, mientras que esta ascendió al 77,9\% (p $<0,01)$ cuando utilizaron las radiografías con tracción y rotación interna.

Por último, en una serie consecutiva de 78 pacientes, Khurana y cols. ${ }^{11}$ demostraron que la precisión en la clasificación de las fracturas de fémur proximal aumentó del 44,9\% al 72,4\% al incorporar la radiografía con tracción y rotación interna $(\mathrm{p}<0,001)$.

Resaltamos como principales debilidades de este estudio el diseño retrospectivo y la falta de un análisis estadístico de los resultados.

\section{CONCLUSIONES}

De acuerdo con los resultados obtenidos podemos concluir en que la radiografía con tracción y rotación interna es un estudio simple, de bajo costo y bien tolerado por el paciente. Por lo tanto, debería realizarse, en forma rutinaria, cuando ingresa un paciente con fractura de fémur proximal, ya que facilita la correcta interpretación de las fracturas y esto tiene un impacto directo en la indicación del tratamiento, el implante por utilizar y su resultado final.

Conflicto de intereses: Los autores no declaran conflictos de intereses.

\section{BIBLIOGRAFÍA}

1. Kanis JA, Odén A, McCloskey EV, Johansson H, Wahl DA, Cooper C. A systematic review of hip fracture incidence and probability of fracture worldwide. Osteoporos Int 2012;23:2239-56. https://doi.org/10.1007/s00198-012-1964-3

2. Garden RS. Low-angle fixation in fractures of the femoral neck. J Bone Joint Surg Br 1961;43:647-63. https://doi.org/10.1302/0301-620X.43B4.647

3. Evans EM. The treatment of trochanteric fracture of the femur. J Bone Joint Surg Br 1949;31:190-203. PMID: 18150534

4. Oakes DA, Jackson KR, Davies MR, Ehrhart KM, Zohman GL, Koval J, et al. The impact of the garden classification on proposed operative treatment. Clin Orthop Relat Res 2003;(409):232-40. https://doi.org/10.1097/01.blo.0000059583.08469.e5

5. Wiltse LL. Internal rotation for the diagnosis of impacted fracture of the hip. Clin Orthop Relat Res 1974;(103):20. https://doi.org/10.1097/00003086-197409000-00008

6. Andersen E, Jorgensen LG, Hededam LT. Evans' classification of trochanteric fractures: an assessment of the interobserver and intraobserver reliability. Injury 1990;21(6):377-8. https://doi.org/10.1016/0020-1383(90)90123-c

7. Pervez H, Parker MJ, Pryor GA, Lutchman L, Chirodian N. Classification of trochanteric fracture of the proximal femur: a study of the reliability of current systems. Injury 2002;33(8):713-5. https://doi.org/10.1016/s0020-1383(02)00089-x

8. Gehrchen PM, Nielsen JO, Olesen B. Poor reproducibility of Evans' classification of the trochanteric fracture. Assessment of 4 observers in 52 cases. Acta Orthop Scand 1993;64(1):71-2. https://doi.org/10.3109/17453679308994533

9. Wiltse LL. Internal rotation for the diagnosis of impacted fracture of the hip. Clin Orthop Relat Res 1974;103:20. https://doi.org/10.1097/00003086-197409000-00008

10. Koval KJ, Oh CK, Egol KA. Does a traction-internal rotation radiograph help to better evaluate fractures of the proximal femur? Bull NYU Hosp Jt Dis 2008;66(2):102-6. PMID: 18537778

11. Khurana B, Mandell JC, Rocha TC, Duran-Mendicuti MA, Jimale H, Rosner B, et al. Internal rotation traction radiograph improves proximal femoral fracture classification accuracy and agreement. AJR Am J Roentgenol 2018; 211(2):409-15. https://doi.org/10.2214/AJR.17.19258AJR 2018; 211:1-7 